\section{Erhöht Migräne das Schlaganfallrisiko nach Operation?}

Eine Studie zeigt, dass das Risiko für einen perioperativen Schlaganfall bei Patienten mit Migräne signifikant erhöht ist. Das ist noch kein Grund zur Panikmache: die absoluten Zahlen sind immer noch niedrig und zudem müssen sekundäre Risiken berücksichtigt werden.

$\mathrm{M}$ igräne (mit Aura) geht bei jüngeren Frauen mit einem leicht erhöhten Risiko für einen Schlaganfall einher. Weiterhin weiß man, dass das Risiko für einen Schlaganfall nach operativen Eingriffen an Gefäßen oder am Herzen ebenfalls erhöht ist $(7,4 \%$ in den ersten 30 Tagen). Es stellt sich somit die Frage, ob Migräne auch das Risiko für einen perioperativen Schlaganfall erhöht. Dazu wurden 124.558 Patenten mit einer Operation ermittelt, von denen 10.179 $(8,2 \%)$ Migräne hatten $(1.278=12,6 \%)$ Migräne mit Aura). Insgesamt gab es 771
$(0,6 \%)$ perioperative Schlaganfälle, insbesondere nach Gefäßoperationen (4,0\%), Herzoperationen (3,5\%) und neurochirurgischen Eingriffen $(2,4 \%)$. Von den Schlaganfallpatienten hatten 9,2\% Migräne ohne Aura und 2,3\% Migräne mit Aura. Zusammenfassend war das Risiko für einen perioperativen Schlaganfall bei Patienten mit Migräne signifikant erhöht (adjusted odd ratio 1,75; $95 \%$-KI 1,39-2,21). Die perioperativen Schlaganfälle traten am häufigsten in den ersten beiden Tagen nach der Operation auf $(46,2 \%), 25,3 \%$ traten

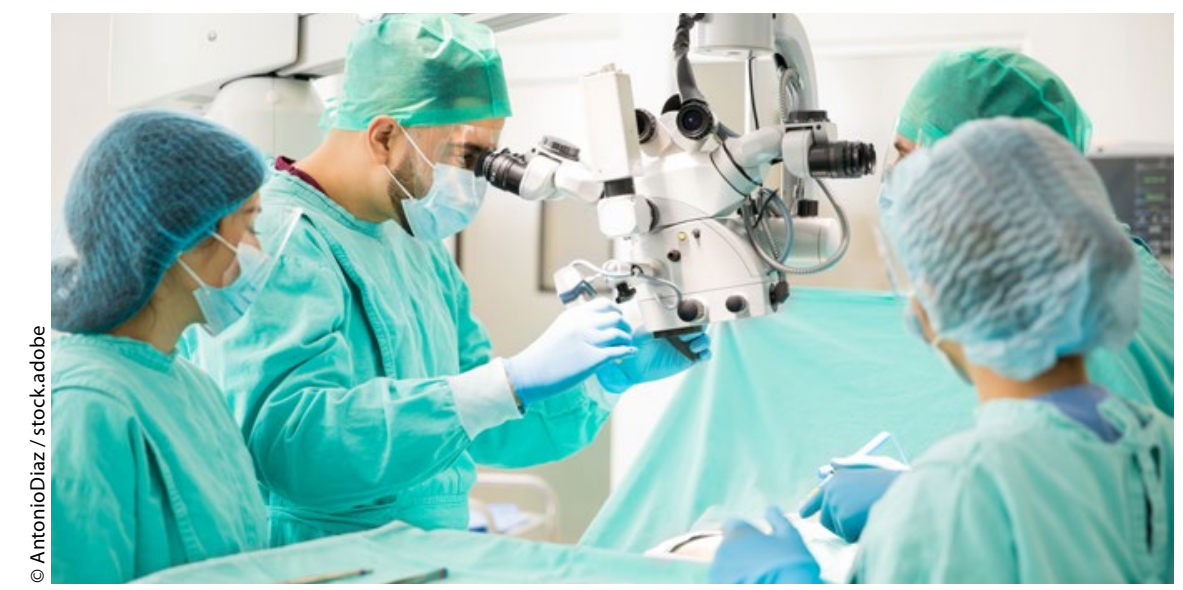

Das Risiko für einen Schlaganfall ist nach operativen Eingriffen an Gefäßen oder am Herzen bereits erhöht - auch ohne Vorliegen einer Migräne. aber auch erst nach Entlassung aus dem Krankenhaus auf. 10.088 Patienten wurden innerhalb von 30 Tagen nach der Operation erneut im Krankenhaus aufgenommen. Auch hier lag das Risiko für Migränepatienten höher (adjusted odd ratio 1,$31 ; 95 \%$-KI $1,22-1,41$ )

\section{Kommentar}

Diese Studie hat in den Medien ein großes Echo hervorgerufen. Leider ist die verkürzte Botschaft, dass Migränepatienten ein erhöhtes perioperatives Risiko für einen Schlaganfall haben, wenig hilfreich. Zum einen muss man sich die absoluten Risiken anschauen; die sind nämlich sehr niedrig: Von 1.000 Operierten erleiden insgesamt $2,4(95 \%-K I 2,1-2,8)$ einen perioperativen Schlaganfall. Von 1.000 Operierten mit Migräne sind dies 4,2 (95\%-KI 3,2-5,3). Außerdem muss berücksichtigt werden, ob sekundäre Risiken vorliegen. Wir haben zum Beispiel perioperativ ohnehin eine erhöhte Rate von Migräneattacken und somit eine erhöhte Rate von Medikamenteneinnahmen (wobei Triptane und Ergotamine nicht ausgeschlossen werden können). Außerdem nehmen manche Migränepatienten vasoaktive Substanzen zur Prophylaxe, dies kann auch das Risiko für einen Schlaganfall erhöhen. Schließlich muss auch die Diagnose Schlaganfall kritisch hinterfragt werden. In den US-amerikanischen Datenbänken wird nach ICD-9 klassifiziert, mögliche TIA oder Minor Strokes ohne Bildbegung können auch einer Migräneaura entsprochen haben, die ebenfalls perioperativ gehäuft auftreten kann.

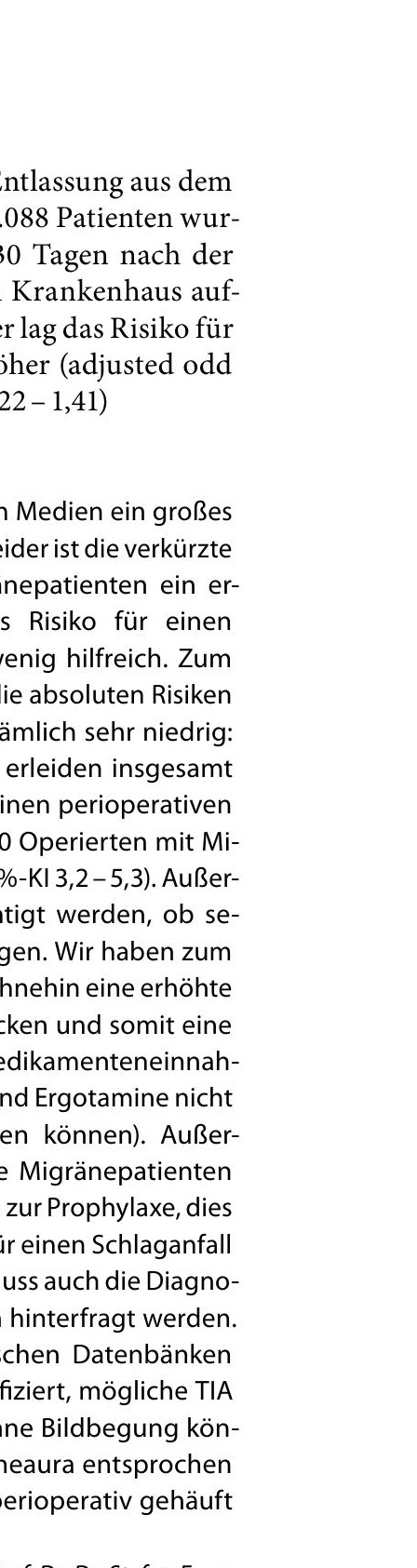

Timm FP et al. Migraine and risk of perioperative ischemic stroke and hospital readmission: hospital based registry study. BMJ 2017; 356: i6635 press 